\title{
A simplex method for the calibration of a MEG device
}

\author{
Valentina Vivaldi ${ }^{1,2}$, Sara Sommariva ${ }^{2}$ Alberto Sorrentino ${ }^{2 *}$ \\ ${ }^{1}$ Istituto di Cibernetica, CNR, Napoli, Italy \\ ${ }^{2}$ Dipartimento di Matematica, Facoltà di Scienze MM.FF.NN. \\ Università degli Studi di Genova, Genova, Italy \\ *Email address for correspondence: sorrentino@dima.unige.it \\ Communicated by Michele Piana and Luigi Preziosi \\ Received on 11 30, 2016. Accepted on 11 13, 2017.
}

\begin{abstract}
MagnetoEncephaloGraphy (MEG) devices are helmet-shaped arrays of sensors that measure the tiny magnetic fields produced by neural currents. As they operate at low temperature, they are typically immersed in liquid helium. However, during the cooling process the sensor position and shape can change, with respect to nominal values, due to thermal stress. This implies that an accurate sensor calibration is required before a MEG device is utilized in either neuroscientific research or clinical workflow. Here we describe a calibration scheme developed for the optimal use of a MEG system recently realized at the "Istituto di Cibernetica e Biofisica" of the Italian CNR. To achieve the calibration goal a dedicated magnetic source is used (calibration device) and the geometric parameters of the sensors are determined through an optimisation procedure, based on the Nelder-Mead algorithm, which maximises the correlation coefficient between the predicted and the recorded magnetic field. Then the sensitivity of the sensors is analytically estimated. The developed calibration procedure is validated with synthetic data mimicking a real scenario.
\end{abstract}

Keywords: MagnetoEncephaloGraphy, Calibration, Calibration Device, Nelder-Mead.

AMS subject classification: $65 \mathrm{~K} 10,90 \mathrm{C} 26$

\section{Introduction.}

MagnetoEncephaloGraphy (MEG), [1,2], is a non-invasive brain imaging technique which is able to record the weak magnetic fields $\left(<10^{-10} \mathrm{~T}\right)$ produced outside the scalp by the neural currents that flow inside the brain, with very high temporal resolution (of the order of few milliseconds) and good spatial resolution (of the order of the centimeter). This outstanding temporal resolution, matched only by ElectroEncephaloGraphy (EEG), makes MEG a suitable tool to investigate the neural dynamics in both normal and pathological conditions. To this end, it is crucial to obtain reliable reconstructions of the brain activity from the recorded magnetic field; this, in turn, requires accurate knowledge of the geometry of the device.

A modern MEG device consists of a helmet-shaped array of a few hundreds of SQUID (Superconducting Quantum Interference Device) sensors [3], which are the only ones able to measure the tiny magnetic fields produced by the brain. In order to maintain the superconductivity, they need to operate within a few degrees from absolute zero (i.e. 4.2 Kelvin), and are therefore immersed in liquid helium. The parameters that describe the geometry of the sensors (position, normal vector and sensitivity) are known at room temperature, at which the MEG-helmet is built, but they are typically modified by thermal stress during the cooling operation. Direct measurements of the final geometric parameters are not possible, as the sensors are immersed in liquid helium inside the dewar and are not visible. Therefore the actual sensor parameters have to be recovered through an accurate calibration procedure.

In literature some calibration methods for multichannel MEG systems have been described so far [4-9]; in this paper we present a detailed description of the algorithm, based on [7], that we have used to calibrate the MEG system [10] developed by the "Istituto di Cibernetica e Biofisica" of the National Research Council, and recently installed in the Magnetoencephalography Laboratory inside the clinic 
"Casa di Cura Hermitage s.p.a." in Naples. Our calibration procedure makes use of a calibration device (calibrator) containing 32 coils (sources) of known positions and orientations. After the system has been cooled down, the calibrator coils are sequentially activated and their magnetic field is measured by the MEG sensors. Clearly, the measured field depends on the geometry of the sensors. Hence, the non-linear optimization algorithm known as Nelder-Mead [11,12] is used to reconstruct the actual parameters by maximizing the correlation between the measured and the predicted magnetic field.

The paper is organised as follows: in Section 2 we introduce the main notations that we use throughout the paper and we describe how we parameterise unknowns and data of the calibration problem; in Section 3 we describe the algorithm we have developed and in Section 4 we present the results obtained with different simulated data. Our conclusions are offered in Section 5.

\section{Methods: notations and models.}

Calibration is a preliminary procedure aimed at providing an accurate estimate of the geometry of the MEG device after the cooling operation, in order to guarantee an efficient use of a multichannel MEG system.

The parameters that describe the geometry of each sensor (position, normal vector and sensitivity) are known at room temperature, but after the necessary immersion in liquid helium they may change, because of thermal stress. In Figure 1 an example of sensor displacement is presented: red stars represent the position of the sensors at room temperature, blue circles represent sensor locations at low temperature.

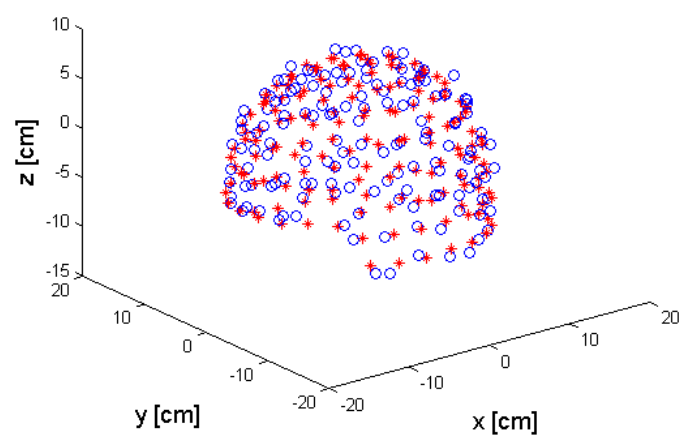

Figure 1. MEG-helmet with both sensors at room temperature (red star) and at low temperature (blue circle).

As the MEG helmet is located inside the MEG pipe, over the recliner, (Figure 2), a direct measure of the sensor parameters is not feasible. To provide a reliable estimate of their values we use a calibrator, i.e. an object of known geometrical and physical properties, that produces dipolar field patterns from point-like sources. The calibrator is put inside the helmet, approximately in the same location where the subject's head usually is. Then the calibrator coils are activated one at a time, and the corresponding magnetic fields are measured by the MEG sensors. As the magnetic fields produced by the calibrator coils are dipolar fields, it is easy to compute their theoretical value. Discrepancies between the theoretical and the measured values are due to two main sources of error: the inexact knowledge of the sensor parameters, and the inexact knowledge of the calibrator parameters. Then an iterative procedure is launched that repeatedly updates the sensor and the calibrator parameters, starting from their nominal values, until the predicted fields are close enough to the measured fields.

In this section we describe how we parametrize the sensors of the MEG-helmet and the sources of the calibrator, how we deal with the measured magnetic field and how we compute the theoretical magnetic field. Moreover we define the notations used throughout the paper. 


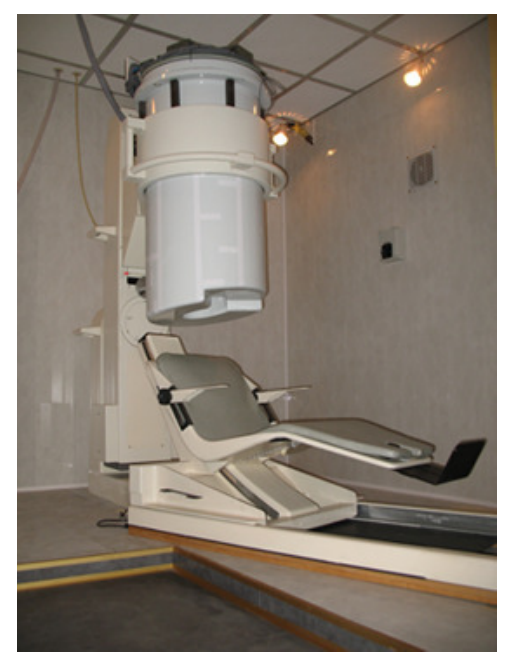

Figure 2. MEG device.

\subsection{Sensors and calibrator parameters.}

Given a coordinate system $X Y Z$ with respect to the helmet, referred to as the Helmet Reference System (HRS) from here on, each sensor $i$ can be completely described by 6 parameters:

- three parameters describe its location, i.e. the cartesian coordinates of its centroid: $\mathbf{r}_{s}(i)=$ $\left(x_{s}(i), y_{s}(i), z_{s}(i)\right)$

- two parameters describe its orientation, i.e. the vector orthogonal to the helmet surface in $\mathbf{r}_{s}(i)$ : $\mathbf{n}_{s}(i)=\mathbf{n}_{s}\left(\theta_{s}(i), \phi_{s}(i)\right)$, where $\theta_{s}(i)$ and $\phi_{s}(i)$ are spherical coordinates;

- one parameter represents the sensitivity, $S(i)$, a multiplicative factor for the magnetic field which takes into account that the area of the sensor may change due to thermal contractions.

In the following, the parameter values before the cooling procedure (nominal values) are denoted with a superposed (0).

The calibrator consists of $N_{\text {coil }}(=31)$ coils regularly placed on a sphere: their locations correspond to the corners of a dodecahedron and its reciprocal icosahedron (Figure 3); their orientation is perpendicular to the sphere surface at that point. In the HRS coordinate system the calibrator can be equivalently

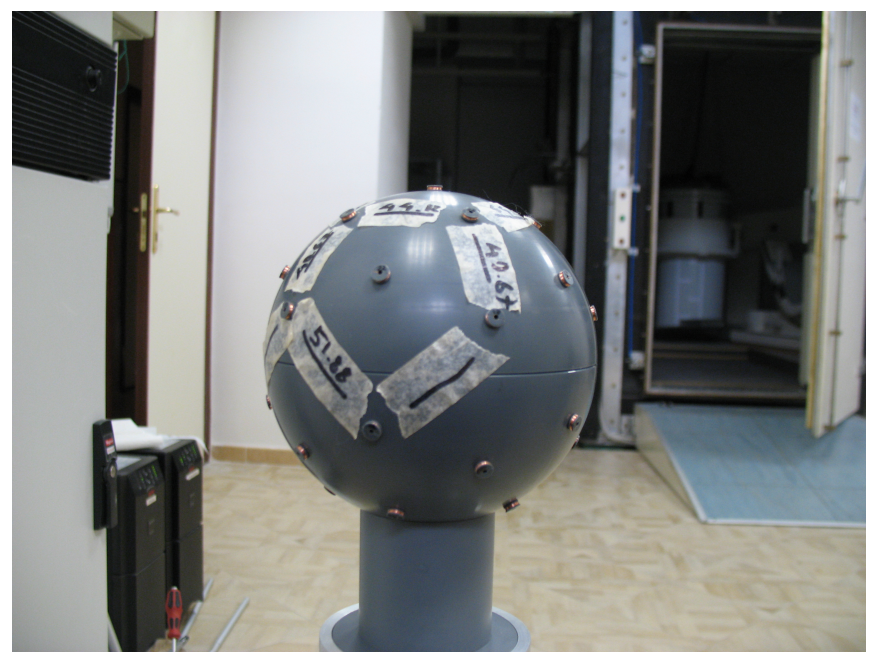

Figure 3. Our calibration device.

described defining the geometry of the sphere as well as defining the parameters of each coil. In the first 
case we need 6 parameters, i.e. the cartesian coordinate of its center $\left(x_{s p h}, y_{s p h}, z_{s p h}\right)$ and three Euler Angles for its orientation $(\alpha, \beta, \gamma)$. Whereas, for each coil $k$ we need:

- three parameters for its position $\mathbf{r}_{c}(k)=\left(x_{c}(k), y_{c}(k), z_{c}(k)\right)$,

- two parameters for its orientation $\mathbf{n}_{c}(k)=\mathbf{n}_{c}\left(\theta_{c}(k), \phi_{c}(k)\right)$,

- one parameter for the dipole intensity $I(k)$, which is proportional to the electrical current flowing in the coil, and to the area of the coil.

Note that the positions and normal vectors of the coils are known in the calibrator coordinate system; once the location and orientation of the calibrator are known in the HRS system, also the coil positions and orientations can be expressed in the HRS. Let us finally remark that the actual values of the coil parameters may differ from nominal values, due to e.g. imperfect construction; the calibration procedure described below accounts for this uncertainty by estimating also the calibration coil parameters.

\subsection{Measured magnetic field.}

The calibrator coils are activated one at a time for a fixed time-interval, typically $60 \mathrm{~s}$, with a sinusoidal current of the form

$$
\Sigma(t)=C \sin \left(2 \pi f\left(t-t_{0}\right)\right)
$$

where $C$ is the amplitude, $f$ is the frequency, $t_{0}$ is the phase.

At the same time, the MEG sensors record the magnetic field produced by the calibrator coils: in the following we indicate by $M_{i, k}(t)$ the magnetic field measured by the $i$-th MEG sensor at time $t$, when current is flowing in the $k$-th coil. We also define the following mean quantity that does not depend on the coil current amplitude $C$ :

$$
M_{i, k}=\frac{\sum_{t=1}^{N_{\text {time }}} \Sigma(t) M_{i, k}(t)}{\sum_{t=1}^{N_{\text {time }}} \Sigma(t)^{2}}
$$

where $N_{\text {time }}$ is the number of time-points.

\subsection{Theoretical magnetic field.}

The exact magnetic field produced by the calibrator can be computed analytically as follows. Each calibrator coil is approximated with a magnetic dipole of moment $\mathbf{Q}^{(0)}(k)=N_{s} \mathbf{n}_{c}^{(0)}(k), N_{s}$ being the number of turns of the coil. Then the magnetic field produced by the $k$-th coil at the location of the $i-$ th MEG sensor is given by

$$
M_{i, k}^{*}=I^{(0)}(k) S^{(0)}(i) \frac{\mu_{0}}{4 \pi}\left(\frac{3 \mathbf{r}^{(0)}(i, k)\left(\mathbf{Q}^{(0)}(k) \cdot \mathbf{r}^{(0)}(i, k)\right)}{r^{(0)}(i, k)^{5}}-\frac{\mathbf{Q}^{(0)}(k)}{r^{(0)}(i, k)^{3}}\right) \cdot \mathbf{n}_{s}^{(0)}(i)
$$

where $I^{(0)}(k)$ is the intensity of dipole $k, S^{(0)}(i)$ is the sensitivity of sensor $i, \mathbf{r}^{(0)}(i, k)=\mathbf{r}_{s}^{(0)}(i)-\mathbf{r}_{c}^{(0)}(k)$, $r^{(0)}(i, k)=\left|\mathbf{r}^{(0)}(i, k)\right|$, and $\cdot$ is the scalar product in $\mathbb{R}^{3}$. For future use, we define

$$
H_{i, k}:=\frac{\mu_{0}}{4 \pi}\left(\frac{3 \mathbf{r}^{(0)}(i, k)\left(\mathbf{Q}^{(0)}(k) \cdot \mathbf{r}^{(0)}(i, k)\right)}{r^{(0)}(i, k)^{5}}-\frac{\mathbf{Q}^{(0)}(k)}{r^{(0)}(i, k)^{3}}\right) \cdot \mathbf{n}_{s}^{(0)}(i)
$$

so that

$$
M_{i, k}^{*}=I^{(0)}(k) S^{(0)}(i) H_{i, k} \quad .
$$




\section{Algorithm.}

The calibration algorithm is an iterative procedure that updates repeatedly the MEG sensor parameter values, until the magnetic field predicted by equation 5 is close enough to the measured data. Because of the non-linearities involved, a good initial guess is necessary to avoid that the algorithm stops in a local minimum.

In this section we first describe how we initialise the unknown parameters, and then we focus on a detailed description of each step of the algorithm.

Initialisation of the geometrical parameters of the sensors: we use the nominal values, i.e. the parameters measured at room temperature.

Initialisation of the geometrical parameters of the calibrator: in order to obtain a reasonable initial guess for the location and orientation of the calibrator within the MEG helmet, we proceed as follows.

- Step 1. We first compute a rough estimate of the location for each coil, via a simple least-squares (see for example [13]):

$$
\hat{\mathbf{r}}_{c}(k)=\operatorname{argmin}_{r_{j} \in\left\{r_{1}, \ldots, r_{J}\right\}}\left\{\left\|M_{:, k}(t)-G\left(\mathbf{r}_{j}\right) \hat{Q}\left(\mathbf{r}_{j}\right)\right\|\right\}
$$

where $M_{:, k}(t)$ is the magnetic field produced by the coil $k$ in all the sensors at a fixed time-point $t$, that is typically chosen as the time-point in which the magnetic field, produced by the coil under examination, has a peak; $\hat{Q}\left(\mathbf{r}_{j}\right)=G\left(\mathbf{r}_{j}\right)^{\dagger} M_{:, k}(t)$ is the estimated dipole strength in $\mathbf{r}_{j} ;\left\{\mathbf{r}_{j}\right\}_{j=1}^{J}$ is the set of the points of a uniform grid built in the space under the sensor-helmet; $G\left(\mathbf{r}_{j}\right)$ is the leadfield-matrix, i.e. a matrix of size $N_{\text {sens }} \times 3$ in which each column contains the magnetic field produced by a unitary magnetic dipole placed in $\mathbf{r}_{j}$ and with dipole moment directed along one of the three orthogonal directions and $\dagger$ is the pseudo-inverse.

- Step 2. We then use a best-fit procedure (Kabsch algorithm [14]) to find the location and orientation of the sphere that bring the nominal coil locations as close as possible to the estimated coil locations.

Initialisation of the intensity and of the sensitivity: an initial value for the intensity of the coils and the sensitivity of the sensors is drawn from an interval whose length is chosen to be the maximal expected displacement between nominal and real values, as observed in real cases.

Iterative optimization procedure: after initialization of all the involved parameters, we start the iterative optimization procedure, aimed at the maximisation of the correlation coefficient between the measured and the predicted magnetic field. Here we use the Nelder-Mead algorithm.

Nelder-Mead is an iterative non-linear optimisation technique: at each iteration the algorithm uses the vertices of a simplex as a set of possible values for the unknowns. The vertices are iteratively changed, by contraction, reflexion or expansion, in order to maximise the functional under study until converge, i.e. until the area of the simplex is less then a give threshold [11].

After having approximated the sphere parameters, we iterate the estimation of the sensors and of the dipoles parameters alternatively, until convergence. In detail, to estimate the geometric parameters of each sensor we maximise, with Nelder-Mead, the correlation coefficient between the measured and the theoretical magnetic field produced by all coils in the fixed sensor. For each dipole we proceed analogously, considering the magnetic field produced by the fixed dipole in all the sensors. On the other hand, the sensitivity and the intensity are calculated through the analytic minimisation of the norm of the difference between the measured and the theoretical magnetic field. For example, the intensity is obtained minimizing the functional $f$ with respect to $I(k)$

$$
f(I(k))=\sum_{i=1}^{N_{\text {sens }}}\left(M_{i, k}-I(k) S(i) H_{i, k}\right)^{2}
$$


where $H_{i, k}$ is the theoretical value of the magnetic field produced by the $k$-th coil and measured by the $i$-th sensor, $S(i)$ is the sensitivity of the $i$-th sensor, $I(k)$ is the intensity of the $k$-th coil and $M_{i, k}$ is the recorded magnetic field.

In a more schematic way, the main steps of our algorithm are:

1) Estimation of the parameters for the calibration sphere. These are six unknowns, $\left(x_{s p h}, y_{s p h}, z_{s p h}, \alpha, \beta, \gamma\right)$, that can be obtained by the maximisation of the correlation coefficient $\rho$ between the measured and the theoretical magnetic field produced by all the coils in all the sensors,

$$
\rho\left(x_{s p h}, y_{s p h}, z_{s p h}, \alpha, \beta, \gamma\right)=\frac{\sum_{i} \sum_{k}\left(M_{i, k}-\bar{M}\right)\left(S(i) I(k) H_{i, k}-\bar{H}\right)}{\sqrt{\sum_{i} \sum_{k}\left(M_{i, k}-\bar{M}\right)^{2} \sum_{i} \sum_{k}\left(S(i) I(k) H_{i, k}-\bar{H}\right)^{2}}}
$$

where

$$
\bar{M}=\frac{\sum_{i} \sum_{k} M_{i, k}}{N_{\text {sens }} N_{\text {coil }}}, \quad \bar{H}=\frac{\sum_{i} \sum_{k} S(i) I(k) H_{i, k}}{N_{\text {sens }} N_{\text {coil }}}
$$

are the mean over all sensors and all dipoles of the measured and of the theoretical magnetic field, respectively. The Nelder-Mead algorithm is used for maximizing $\rho$.

2) Until convergence (that means until larger position displacement between two consecutive iterations is bigger than $1 \mathrm{~mm}$ ) iterate:

For each sensor $i$ :

2a) Estimation of its position and normal vector: these are five unknowns $\left(x_{s}(i), y_{s}(i), z_{s}(i), \theta_{s}(i), \phi_{s}(i)\right)$ that can be obtained by maximisation of the correlation coefficient $F$ between the measured and the theoretical magnetic field, produced by all coils and recorded by the $i$-th sensor;

$$
F\left(x_{s}(i), y_{s}(i), z_{s}(i), \theta_{s}(i), \phi_{s}(i)\right)=\frac{\sum_{k}\left(M_{i, k}-\bar{M}\right)\left(I(k) H_{i, k}-\bar{H}\right)}{\sqrt{\sum_{k}\left(M_{i, k}-\bar{M}\right)^{2} \sum_{k}\left(I(k) H_{i, k}-\bar{H}\right)^{2}}}
$$

where

$$
\bar{M}=\frac{\sum_{k} M_{i, k}}{N_{\text {coil }}}, \quad \bar{H}=\frac{\sum_{k} I(k) H_{i, k}}{N_{\text {coil }}}
$$

are the mean, over all dipoles, of the measured and the theoretical magnetic field, respectively.

2b) Estimation of its sensitivity; this is one unknown $S(i)$ that is given by the analytic formula

$$
S(i)=\frac{\sum_{k}\left(M_{i, k} I(k) H_{i, k}\right)}{\sum_{k} I(k)^{2} H_{i, k}^{2}}
$$

For each coil $k$ :

2c) Estimation

of its position and normal vector; these are five unknowns $\left(x_{c}(k), y_{c}(k), z_{c}(k), \theta_{c}(k), \phi_{c}(k)\right)$ that can be obtained by maximisation of the correlation coefficient $F$ between the measured and the theoretical magnetic field produced by all sensors in the fixed $k$-th coil

$$
F\left(x_{c}(k), y_{c}(k), z_{c}(k), \theta_{c}(k), \phi_{c}(k)\right)=\frac{\sum_{i}\left(M_{i, k}-\bar{M}\right)\left(S(i) H_{i, k}-\bar{H}\right)}{\sqrt{\sum_{i}\left(M_{i, k}-\bar{M}\right)^{2} \sum_{i}\left(S(i) H_{i, k}-\bar{H}\right)^{2}}}
$$

where

$$
\bar{M}=\frac{\sum_{i} M_{i, k}}{N_{\text {sens }}}, \quad \bar{H}=\frac{\sum_{i} S(i) H_{i, k}}{N_{\text {sens }}}
$$

are the mean, over all dipoles, of the measured and the theoretical magnetic field, respectively. 
2d) Estimation of its intensity; this is one unknown $I(k)$ that is given by the analytic formula

$$
I(k)=\frac{\sum_{i}\left(M_{i, k} S(i) H_{i, k}\right)}{\sum_{i} S(i)^{2} H_{i, k}^{2}}
$$

\section{Validation with synthetic data.}

In this section simulated data are used to validate the calibration procedure. We first describe the generation of synthetic data, then show our results.

\subsection{Simulation of the magnetic field.}

Mimicking a real scenario, we simulate an helmet system by assigning values to

- position and orientation of the calibration sphere, i.e. the Cartesian coordinates of the centre $\left(x_{s p h}, y_{s p h}, z_{s p h}\right)$ and the Euler angles $(\alpha, \beta, \gamma)$;

- position, normal vector and intensity of each coil of the calibration sphere $\left\{\mathbf{r}_{c}(k), \mathbf{n}_{c}(k), I(k)\right\}_{k=1}^{N_{c o i l}}$. All coils are radially oriented and their positions with respect to the sphere coordinate system are usually known with good accuracy, thus, as done in [8] we assume them perfectly known. We set $I(k)=1 \forall k$;

- position, normal vector and sensitivity of each sensor $\left\{\mathbf{r}_{s}(i), \mathbf{n}_{s}(i), S(i)\right\}_{i=1}^{N_{\text {sens }}}$.

1. For the position of each sensor, we draw a point with a uniform distribution within a cube centred in the nominal value and with side $\delta_{r}$. In the simulations below $\delta_{r}=2 \mathrm{~cm}$.

2. For the normal vector, we corrupt every spherical coordinate, $\theta_{s}(i)$ and $\phi_{s}(i)$, drawing a value with uniform distribution within the interval centred in the nominal value and with length $\delta_{\theta}$ and $\delta_{\phi}$ respectively. In the simulations below $\delta_{\theta}=\delta_{\phi}=\frac{\pi}{8}$.

3. The sensitivity of each sensor $i$ is defined as $S(i)=1+u$ where $u$ is drawn from a uniform distribution in $[-0.05 ; 0.05]$.

In the following we refer to these parameters, that simulate the parameters at cryogenic temperature, as exact values (Figure 6). We use them to simulate, for each coil, the magnetic field recorded by the sensors when the coil is activated with a current of the form described by equation (1), with $C=10^{-6}, f=12.5$, $t_{0}=0$. The magnetic field (equation (5)) is computed approximating each coil with a magnetic dipole, and using the exact value for the parameters of both sensors and dipoles. We add white Gaussian noise with different standard deviations, in order to obtain data with different levels of noise (including the noise-free case) (Figure 6), and then we average these data over time (equation (2)) to simulate $M_{i, k}$.
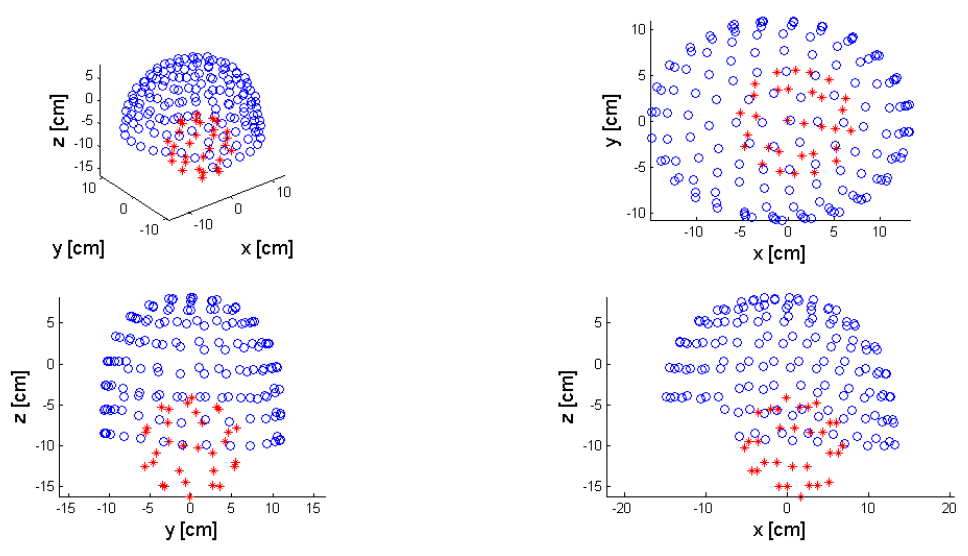

Figure 4. Our nominal values. The blue circles represent the positions of each sensor and the red stars represent the positions of each coil at room temperature. 

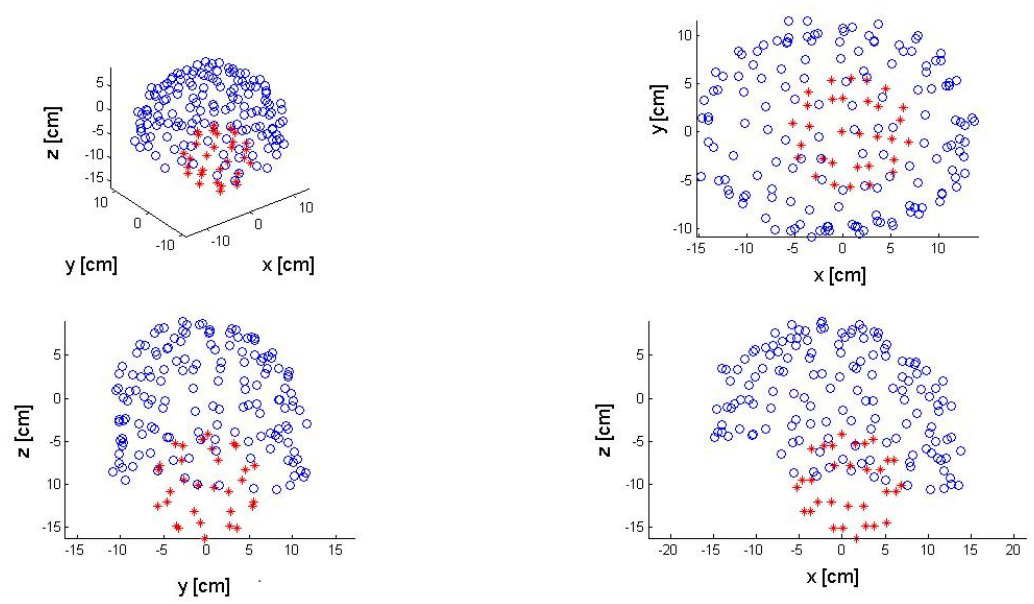

Figure 5. Our exact values. The blue circles represent the positions of each sensor and the red stars represent the positions of each coil at cryogenic temperature.
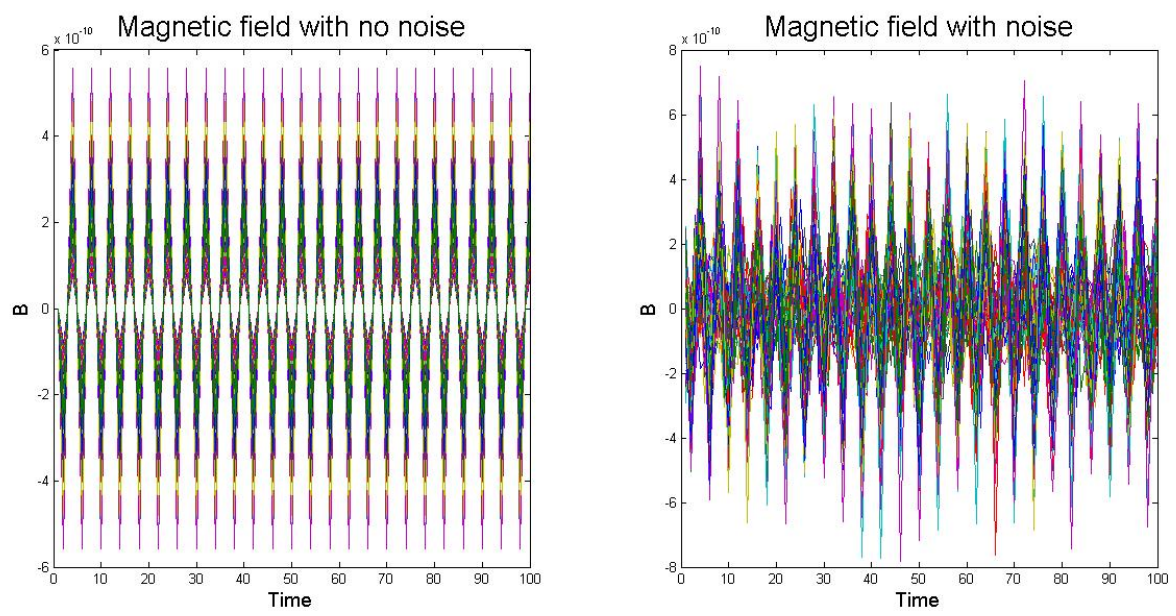

Figure 6. Butterfly plot of the magnetic field produced by a fixed coil before (left) and after (right) adding white Gaussian noise.

\subsection{Results.}

We run the calibration algorithm on the simulated magnetic field with different levels of noise, as described in section 4.1. Now let $\left\{\widehat{\mathbf{r}}_{s}(i), \widehat{\mathbf{n}}_{s}(i), \widehat{S}(i)\right\}_{i=1}^{N_{s e n s}}$ be the set of reconstructed positions, normal vectors and sensitivities of the sensors; for each sensor $i$ we define:

- localisation error:

$$
\Delta_{r}(i)=\left|\widehat{\mathbf{r}}_{s}(i)-\mathbf{r}_{s}(i)\right|
$$

- orientation error:

$$
\Delta_{n}(i)=\arccos \left(\widehat{\mathbf{n}}_{s}(i) \cdot \mathbf{n}_{s}(i)\right)
$$

where $\Delta_{n}(i)$ represents the angle between the exact and the reconstructed normal vector;

- sensitivity error:

$$
\Delta_{S}(i)=\frac{|\widehat{S}(i)-S(i)|}{S(i)}
$$


Importantly, we point out that the optimisation procedure can only estimate the relative positions of sensors and coils, i.e., it works up to a change of reference system. Therefore, to quantify the errors on the estimated parameters, we need to apply a roto-translation, that brings the parameters of the calibration sphere to the true ones. Thus we compute

$$
\widehat{\Delta}_{r}=\left|\widehat{\mathbf{r}}_{s}^{(r t)}(i)-\mathbf{r}_{s}(i)\right|
$$

where $\widehat{\mathbf{r}}_{s}^{(r t)}(i)$ is obtained as the action of the roto-translation on the reconstructed position $\widehat{\mathbf{r}}_{s}(i)$. Similarly we compute $\hat{\Delta}_{n}$.

Similar measures can be computed for the dipoles, defining the reconstructed parameters as $\left\{\widehat{\mathbf{r}}_{c}(k), \widehat{\mathbf{n}}_{c}(k), \widehat{I}(k)\right\}_{k=1}^{N_{c o i l}}$.

In Figure 7 we present results obtained with noiseless data. In the first row we show the exact position of the sensors (blue circles) and, with the red stars, the reconstructed values at the beginning (initialization, left column) after the optimization procedure (middle column) and after the roto-traslation (right column). In the second row we plot the corresponding localization error: as expected, the localisation error after roto-translation $\widetilde{\Delta}_{r}$ is significantly lower then that before the roto-translation $\Delta_{r}$, and remains below $4 \mathrm{~mm}$ for all the sensors.

In Figure 8 and 9 we show the errors on orientation and sensitivity, respectively, that are pleasantly small.
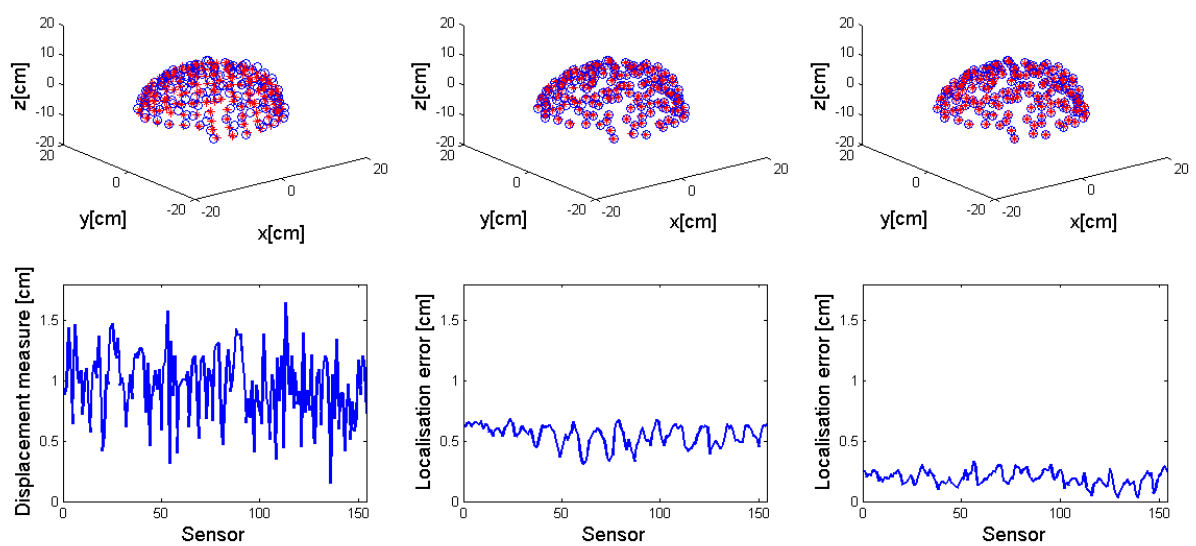

Figure 7. Noiseless data: localisation error. In the first column we plot the initial positions of the sensors and their displacement measure; in the second column we plot the reconstructed positions and the localisation error $\Delta_{r}$ and in the third one we plot the positions reconstructed after the roto-translation and the localisation error $\hat{\Delta}_{r}$. We show the exact position of the sensors with blue circles and the reconstructed ones with red stars.

In Figure 10, 11 and 12 we plot the same quantities in the case of noisy data (standard deviation $\sigma=10^{-10}$ ). The graphs show that the algorithm works fine for most of the sensors except for few ones in which both the localisation error $\Delta_{r}$ and $\widetilde{\Delta}_{r}$ become very high. To investigate this issue, we computed the relative SNR for each sensor, and confirmed that the localization error is large for sensors that have an extremely low SNR.

\section{Conclusions.}

In this paper we have described an algorithm for the calibration of a MEG helmet system, based on the algorithm described by Pasquarelli et al. in [6] and [7]. Recently more sophisticated algorithms have been presented for example in [8], but we chose the one of Pasquarelli because of its efficiency and simplicity: future work may regard a comparison between these two approaches. 

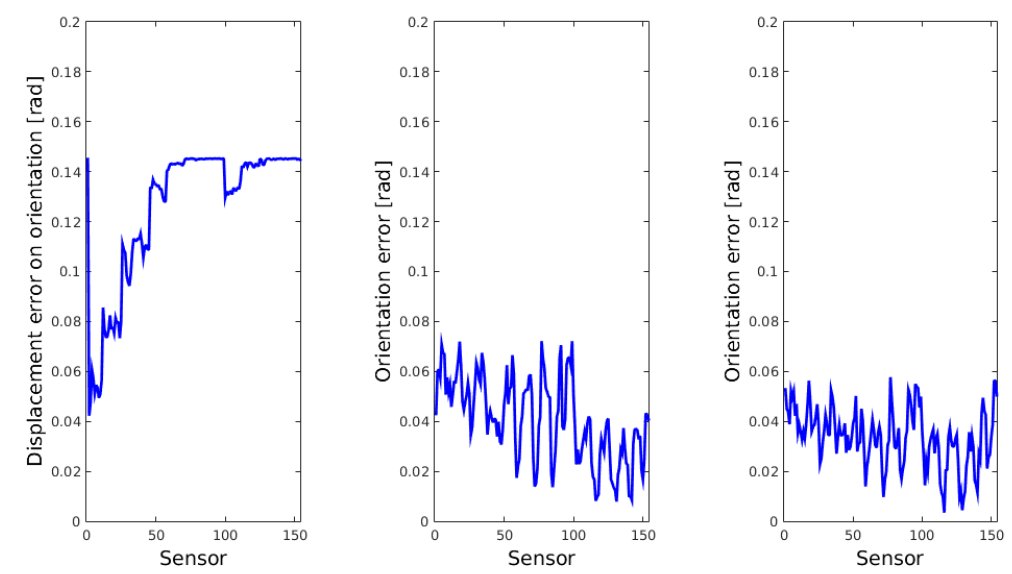

Figure 8. Noiseless data: orientation error. In the first column we plot the initial orientation displacement, in the second column the orientation error $\Delta_{n}$ and in the third one the orientation error after the roto-translation $\hat{\Delta}_{n}$.

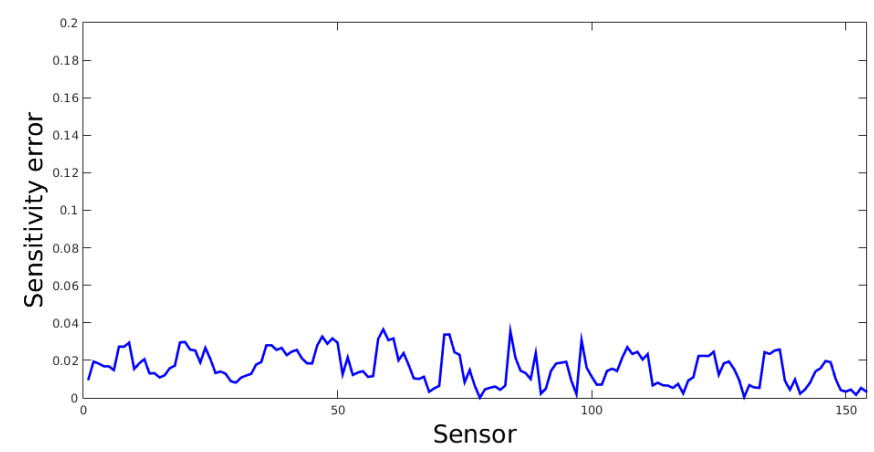

Figure 9. Noiseless data: sensitivity error.
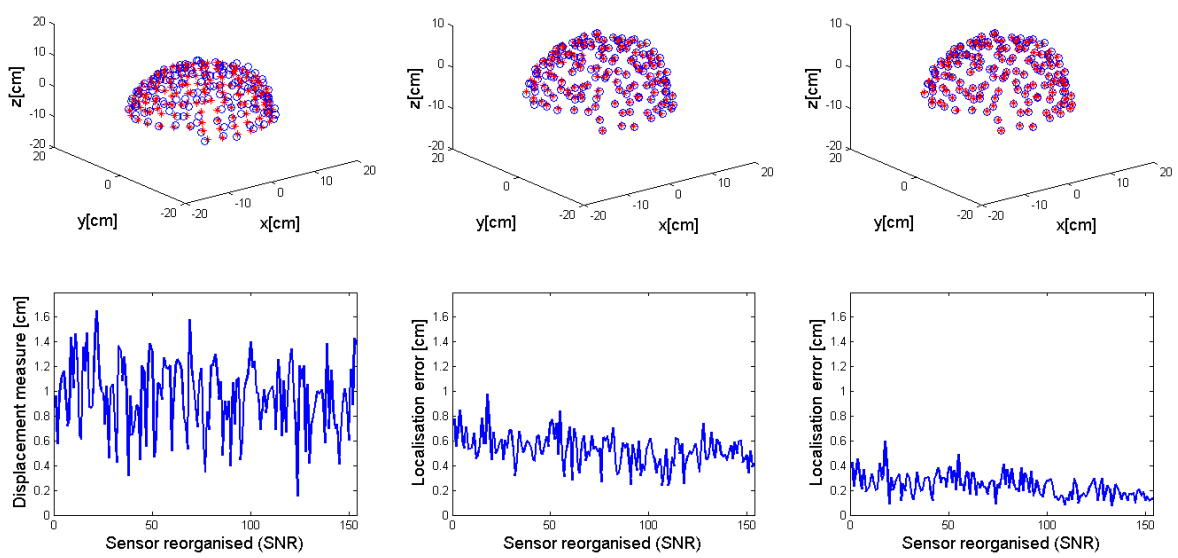

Figure 10. Noisy data: localisation error. In the first column we plot the initial positions of the sensors and their displacement measure; in the second column we plot the reconstructed positions and the localisation error $\Delta_{r}$ and in the third one we plot the positions reconstructed after the roto-translation and the localisation error $\hat{\Delta}_{r}$. We show the exact position of the sensors with blue circles and the reconstructed ones with red stars.

More in detail, our procedure makes use of a specifically constructed calibration device, which produces known magnetic fields. Then the geometric parameters that define position and orientation of the sensors 

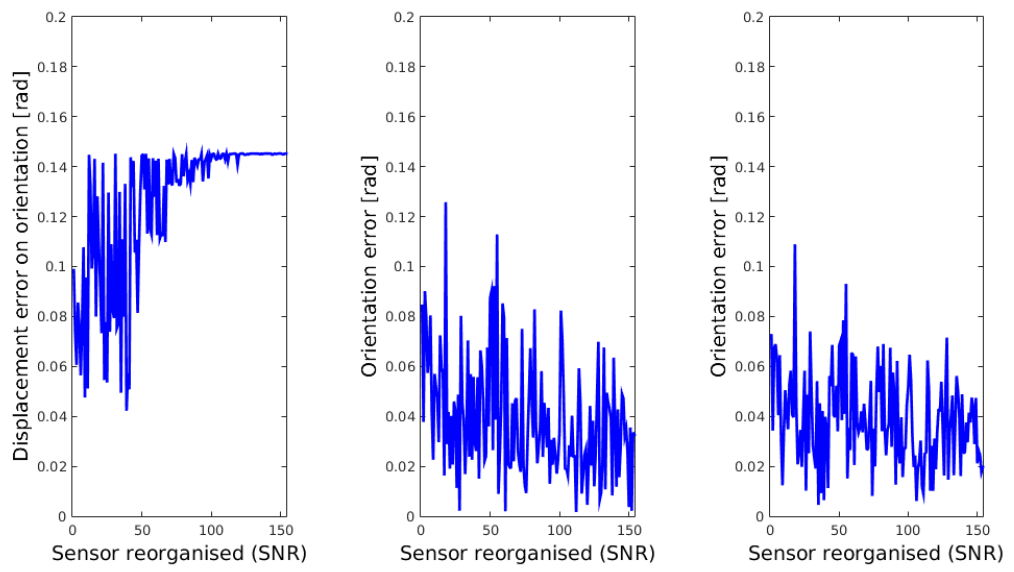

Figure 11. Noisy data: orientation error. In the first column we plot the initial orientation displacement, in the second column the orientation error $\Delta_{n}$ and in the third one the orientation error after the roto-translation $\hat{\Delta}_{n}$.

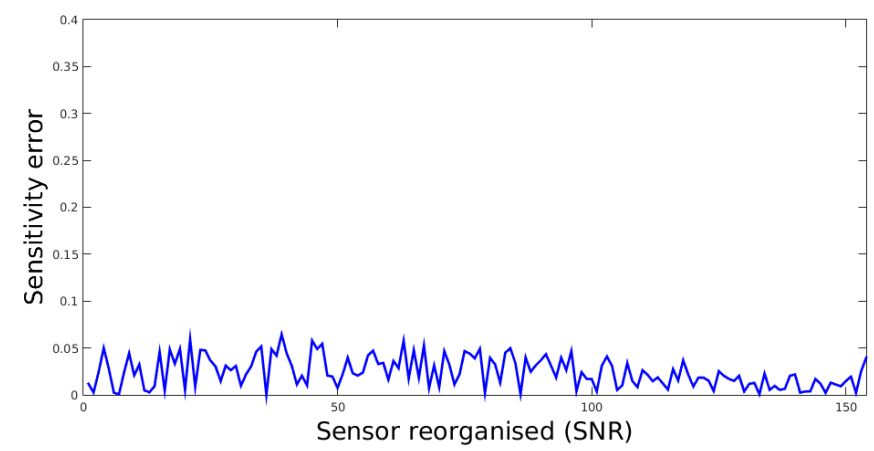

Figure 12. Noisy data: sensitivity error.

are determined through maximisation of the correlation coefficient between the predicted and the recorded magnetic field. The sensitivity of the sensors is estimated analytically.

We have validated our method with noiseless and noisy data. The results we obtained look satisfactory: for example, in the noiseless case, the sensors location parameters are reconstructed up to a roto-translation with a localization error which remains under $4 \mathrm{~mm}$ for all the sensors, whereas, with noisy data, we have verified that the sensors with higher reconstruction error are the ones with lower SNR.

Future work will concern the application of the described algorithm to real data.

\section{Acknowledgements}

The authors kindly acknowledge the Gruppo Nazionale per il Calcolo Scientifico and Istituto di Cibernetica 'Eduardo Caianiello' for financial support. Sara Rombetto, Ruggero Rossi are kindly acknowledged for their technical support in the analysis of the data.

\section{References}

1. M. Hämäläinen, R. Hari, J. Knuutila, and O. Lounasmaa, Magnetoencephalography: theory, instrumentation and applications to non-invasive studies of the working human brain, Reviews of Modern Physics, vol. 65, pp. 413-498, 1993.

2. C. Del Gratta, V. Pizzella, F. Tecchio, and G. Romani, Magnetoencephalography-a noninvasive brain imaging method with $1 \mathrm{~ms}$ time resolution, Reports on Progress in Physics, vol. 64, pp. 1759-1814, 2001. 
3. C. P. Foley, M. N. Keene, H. J. M. ter Brake, and J. Vrba, Squid system issues. Wiley-VCH Verlag GmbH \& Co KGaA, Weinheim, 2004.

4. A. Bruno and P. C. Ribeiro, Spatial fourier calibration method for multichannel squid magnetometers, Review of scientific instruments, vol. 62, pp. 1005-1009, 1991.

5. J. R. Kraus, A. Matlacov, P. Volegov, M. Espy, K. Maharajh, and E. Flynn, Source localization precision of the superconducting imaging-surface meg system, Biomedizinische Technik/Biomedical Engineering, vol. 46, pp. 38-40, 2001.

6. A. Pasquarelli, R. Rossi, M. D. Melis, L-Marzetti, A. Trebeschi, H. Muller, and S. Ernè, Argo 500: Operation of a helmet vector-meg, Neurology and Clinical Neurophysiology, vol. 97, p. : 16012682, 2004.

7. A. Pasquarelli, R. Rossi, M. D. Melis, L-Marzetti, A. Trebeschi, H. Muller, and S. Ernè, Calibration of a vector-meg helmet system, Neurology and Clinical Neurophysiology, vol. 94, p. : 16012682, 2004.

8. F. Chella, F. Zappasodi, S. D. Penna, and V. Pizzella, Calibration of a multichannel MEG system based on the Signal Space Separation method, Physics in Medicine and Biology, vol. 57, pp. 48554870, 2012.

9. C. Wienbruch, Meg-calibration- v. 2.0, tech. rep., Department of Psychology, University of Konstanz, Germany, 2013.

10. A. V. S. Rombetto, C. Granata and M. Russo, Multichannel system based on a high sensitivity superconductive sensor for magnetoencephalography, Sensors, vol. 14 (7), pp. 12114-12126, 2014.

11. J. A. Nelder and R. Mead, A simplex method for function minimization, Computer Journal, vol. 7 (4), pp. 308-313, 1965.

12. R. O'Neill, Function minimization using a simplex procedure, Journal of the Royal Statistical Society. Series C (Applied Statistics), vol. 20 (3), pp. 338-345, 1971.

13. S. Baillet, J. Mosher, and R. Leahy, Electromagnetic brain mapping, Signal Processing Magazine, IEEE, vol. 18, pp. 14-30, 2001.

14. W. Kabsch, A solution for the best rotation to relate two sets of vectors, Acta Crystallographica, vol. 32, pp. 922-923, 1976. 